\title{
Interpretation of Micelle Diffusion Coefficients
}

\author{
GEORGE D. J. PHILLIES \\ Department of Chemistry. The University of Michigan, Ann Arbor, Michigan 48109
}

Received May 4, 1981; accepted July 9, 1981

\begin{abstract}
The interpretation of micelle diffusion coefficients, especially those obtained by means of quasielastic light-scattering spectroscopy (QELSS), is discussed in light of theoretical work on macroparticle diffusion. The alleged discrepancy between QELSS results for $D$ and results obtained by tagging micelles with dye arises because the experiments measure different physical parameters. Possible influences of monomer-micelle equilibria on QELSS data are examined, taking into account the distinction between those reactions which actually change the number of micelles and those reactions which only modulate the aggregation number $n$ of individual micelles.
\end{abstract}

\section{INTRODUCTION}

The objective of this paper is to compare recent and classical experiments on the diffusion of surfactant micelles with modern theories, based on statistical mechanics, of macroparticle transport in solution. The fundamental theoretical results discussed here are, with rare exceptions, not new; the novelty is in their application to the behavior of micelles rather than rigid macroparticles. Specifically novel is the consideration of monomer-micelle equilibria, as given by the work of Aniansson et al. (1), and its effect on quasi-elastic light-scattering spectra. By using modem interpretations, several outstanding problems involving micelle diffusion are resolved; in particular, it is argued here that the alleged (2) discrepancy between the diffusion coefficient measurements of Stigter et al. (3) and of Corti and Degiorgio (4-6) does not exist.

There is available a substantial body of precise experimental data on micelle diffusion; as discussed below, their interpretation has sometimes relied on models more simple than the physical system would appear to require. This note begins by presenting the modern microscopic models for diffusion of interacting particles, developed largely for use with quasi-elastic lightscattering spectroscopy (QELSS). The experimental data on diffusion of SDS micelles are shown to be in agreement with the better theoretical work. The influence of micellemonomer kinetics on QELSS spectra is then examined. The effect of monomer-micelle reactions on the average linewidth is argued to be slight; however, the kinetic effects probably make it very difficult to determine micelle polydispersity from the spectral lineshape. While previous papers on QELSS experiments have noted the possibility of kinetic effects, the discussion here is the first to use a fully developed model (1) for micelle kinetics.

\section{THEORETICAL RESULTS ON DIFFUSION}

Two general sorts of experiment exist for measuring translational diffusion coefficients of macroparticles. In one scheme, one sets up a gradient in the solute concentration, and then observes the disappearance of the gradient with time; the diffusion coefficient obtained in this way is the mutual (or pair) diffusion coefficient $D_{\mathrm{m}}$. In the other scheme, one takes a solution with a uniform solute concentration, selectively labels some of the solute molecules (while leaving the total solute concentration constant), and meas- 
ures the motion of the labeled solute particles through the background of unlabeled particles. The motion of the labeled particles is determined by the tracer (or singleparticle) diffusion coefficient $D_{\mathrm{T}}$.

Extensive studies exist of both $D_{\mathrm{T}}$ and $D_{\mathrm{m}}$; for example, inelastic neutron scattering can measure either quantity. $D_{\mathrm{T}}$ and $D_{\mathrm{m}}$ measure distinct physical processes, and in general do not have the same concentration dependence (7). The classical analysis allows one to write (8)

$$
\begin{aligned}
D_{\mathrm{T}} & =\frac{K_{\mathrm{B}} T}{f_{\mathrm{t}}}, \\
D_{\mathrm{m}} & =\frac{\left(\frac{\partial \pi}{\partial c}\right)_{T, \mu}(1-\phi)}{f_{\mathrm{m}}},
\end{aligned}
$$

but these forms are not adequate for diffusion over very short distances (9). Here $K_{\mathrm{B}} T$ is the thermal energy, $(\partial \pi / \partial c)_{\mathrm{T}, \mu}$ is the osmotic compressibility of the solute, $\phi$ is the solute volume fraction, and $f_{\mathrm{m}}$ and $f_{\mathrm{t}}$ are the drag coefficients for the macroparticles in the two cases. Physically, [1a] and [1b] differ because the environments of the diffusing particles are different. In tracer diffusion, each particle observes the same total density of other macroparticles in all directions, so that the thermal-average interparticle force $\langle F\rangle$ on the particle of interest vanishes. In mutual diffusion, the concentration gradient creates a preferred direction in space along which $\langle\mathbf{F}\rangle$ can point.

$f_{\mathrm{m}}$ and $t_{\mathrm{t}}$ both depend on $c$, but it is not agreed whether or not $f_{\mathrm{m}}$ and $f_{\mathrm{t}}$ are equal. Experimental data on protein solutions suggest their approximate equality (10). There is an ongoing theoretical dispute over the role of "dynamic friction" - the enhancement of $f$ by direct interactions (i.e., collisions). Several authors $(11-13)$ have claimed that dynamic friction contributes to $f_{\mathrm{t}}$ but not to $f_{\mathrm{m}}$, at least in the long-wavelength limit, while this author has presented a microscopic calculation which appears to show that dynamic friction does contribute to $f_{\mathrm{m}}$ (14). Since Mazo (15) has demonstrated that dynamic friction can have a large effect on $D_{\mathrm{T}}$ in low-salt micelle preparations, this disagreement is not inconsequential. The dispute does not affect the theoretical consensus that $D_{\mathrm{T}}$ and $D_{\mathrm{m}}$ are unequal.

The accuracy of [1a] and [1b] for predicting macroparticle diffusion has been most extensively tested on solutions of proteins, notably hemoglobin and serum albumin. It is found that $D_{\mathrm{T}}$ decreases sharply with increasing protein concentration. For hemoglobin, $D_{\mathrm{T}}$ ranges from $\sim 8 \times 10^{-7} \mathrm{~cm}^{2} \mathrm{sec}^{-1}$ at low concentration to $\sim 5 \times 10^{-7} \mathrm{~cm}^{2} \mathrm{sec}^{-1}$ at $100 \mathrm{~g} \mathrm{liter}^{-1}$ and $1.7 \times 10^{-7} \mathrm{~cm}^{2} \mathrm{sec}^{-1}$ at $300 \mathrm{~g} \mathrm{liter}^{-1}$ (16). Between 0 and $300 \mathrm{~g} \mathrm{liter}^{-1}$, Jones et al. (17) found that $D_{\mathrm{m}}$ of hemoglobin falls by only $16 \%$. Similarly, Phillies et al. (18) found that $D_{\mathrm{m}}$ for serum albumin depends strongly on $\mathrm{pH}$ as well as concentration. $\left(d D_{\mathrm{m}} / d c\right)_{c=0}$ for serum albumin at ionic strength $0.15-0.25$ is sharply positive

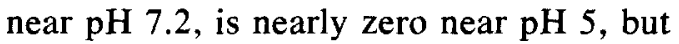
is modestly negative near $\mathrm{pH}$ 4.5. Since serum albumin does not undergo any conformational changes over this $\mathrm{pH}$ range, the change in $\left(d D_{\mathrm{m}} / d c\right)$ with $\mathrm{pH}$ must arise from interactions between the serum albumin molecules.

Elaborate efforts have been made to calculate the concentration dependences of $D_{\mathrm{m}}$ and $D_{\mathrm{T}}$. The concentration dependence of $D_{\mathrm{T}}$ arises from hydrodynamic interactions and dynamic friction. Close to the $\mathrm{CMC}$, the number density of micelles is small, so hydrodynamic interactions become unimportant. However, if the ionic strength of the solvent is low, electrostatic forces between micelles can still be large, so that the concentration dependence of $D_{\mathrm{T}}$ is dominated by dynamic friction. Mazo (15) obtains the formula

$$
\begin{aligned}
\frac{\Delta f}{f_{0}}=-\frac{2 \pi c}{3} \frac{F}{K^{3}} & {\left[\frac{e^{-b}}{b} \int_{0}^{b} x^{2}[g(x)-1] d x\right.} \\
& \left.+\int_{b}^{\infty} x[g(x)-1) d x\right]
\end{aligned}
$$


for the ratio of the change in $f_{\mathrm{t}}$ due to dynamic friction to the single-particle drag coefficient. $F$ gives the interaction strength, $K$ is the inverse Debye length, $b$ is an arbitrary small cut-off length, and $X=K r$.

To calculate $D_{\mathrm{m}}$, one needs expressions for the hydrodynamic interactions and for the interparticle potential. Hydrodynamics is commonly approximated by use of the Oseen tensor and its short-range corrections; the basic Oseen tensor gives the fluid flow due to the motion of a point particle through the fluid. In dilute solution, the interparticle potential of average force reduces to the pair potential. On taking into account direct and hydrodynamic effects, but not dynamic friction, the diffusion coefficient for a suspension of pointlike spherical particles is predicted (to first order in the concentration) to be (19)

$$
\begin{aligned}
D_{\mathrm{m}} & =D_{0}\left[1-\phi+4 \pi c \int_{0}^{\infty} d r r^{2}\right. \\
& \left.\times\left[\frac{a}{r}(g(r)-1)-\frac{5}{2}\left(\frac{a}{r}\right)^{4} g(r)\right]\right] .
\end{aligned}
$$

Here $D_{0}$ is the diffusion coefficient of an isolated particle; $a$ is the sphere radius and $g(r)=\exp \left[-V(r) / K_{\mathrm{B}} T\right]$ is the radial distribution function. There appear in the literature a wide variety of expressions similar (but not identical) to [3] $(11,20,21)$. The variation results from the range of equations which exist for the hydrodynamic interaction of two spheres, not from fundamental physical questions; [3] appears to incorporate the most complete expression for the hydrodynamic interactions, as obtained by Felderhof (20). For micelles, the best available potential is that of Corti and Degiorgio (6), which includes hard-sphere contact, screened electrostatic repulsion, and an attractive van der Waals interaction.

In quasi-elastic light-scattering spectroscopy, one measures the temporal behavior of fluctuations in the intensity of light scattered by a fluid, scattering through each particular pair of directions being due to fluc- tuations in a specific spatial fourier component of the concentration. It can be shown (7) that the spectrum $S(\mathbf{q}, t)$ (as measured in time domain) of the scattered light is related to the positions of the scattering molecules by

$S(\mathbf{q}, t)=B+A\left|\left\langle\sum_{i, j=1}^{N} e^{i \mathbf{q} \cdot\left[\mathbf{r}_{i}(t)-\mathbf{r}_{j}(t+\tau)\right]}\right\rangle\right|^{2}$,

$A$ and $B$ being experimental constants. The rate at which an intensity fluctuation decays back to its average value is determined by the rate of decay of the corresponding sinusoidal concentration fluctuation. While complications may arise because of the short distance over which diffusion occurs (8) or the large size of local concentration fluctuations (22), it is generally agreed that QELSS measures the relaxation of a concentration gradient, i.e., it measures $D_{\mathrm{m}}$, not $D_{\mathrm{T}}$.

In a sufficiently dilute solution, $D_{\mathrm{m}}$ and $D_{\mathrm{T}}$ become experimentally indistinguishable and equal to the isolated particle diffusion coefficient $D$. By using the StokesEinstein equation

$$
D=\frac{K_{\mathrm{B}} T}{6 \pi \eta r}
$$

one may calculate from $D$ the radius $r$ of a hydrodynamically equivalent sphere, but the application of this formula to $D_{\mathrm{m}}$ is only justified in very dilute solutions. In some special cases [serum albumin (18) near $\mathrm{pH} 5$, model solution of hard spheres whose $f_{\mathrm{m}}$ has the same dependence on $c$ as $f_{\mathrm{t}}$ of serum albumin (9)], $D_{\mathrm{m}}$ is nearly independent of concentration. However, these are special cases, not directly comparable to micelle systems. Even when its net charge is zero, serum albumin molecules attract each other strongly via electrostatic dipole-dipole interactions. The hard-sphere solution is a mathematical model system whose component particles lack the van der Waals attractions expected to exist between real particles such as micelles (6). As a result, 
as this author has repeatedly emphasized, QELSS values for $D_{\mathrm{m}}$ in concentrated solution do not, in the absence of substantial bodies of auxiliary data, reveal the effect of concentration on the hydrodynamic radius $r$ of the diffusing macroparticles. Indeed, for the concentrated hard-sphere suspension treated in Ref. (9), changes in $r$ and the corresponding changes in $D_{\mathrm{m}}$ are not always of the same sign, let alone magnitude. Only by correcting $D_{\mathrm{m}}$ for intermicellar interactions, as attempted by Corti and Degiorgio (6) or Rohde and Sackman (23), can $D_{\mathrm{m}}$ in nondilute solutions be interpreted in terms of a macroparticle radius.

\section{EXPERIMENTAL DATA}

Stigter et al. (3) report on the diffusion of labeled SDS micelles through a solution whose total ionic strength and surfactant concentration were everywhere the same. The label was the water-insoluble dye orange OT; to prevent convection, the diffusion was carried out inside fritted glass disks. The use of fritted glass plates of different degrees of coarseness had no effect on the results, arguing that surface effects were not important. These measurements are undoubtedly of tracer (self) diffusion. $D_{T}$ falls as $c$ is increased. The $c$ dependence was calculated by Mazo (15), using electrophoretic data of Stigter and Mysels to estimate the intermicellar potential. Reasonable numerical agreement is found with the observation that reducing the $\mathrm{NaCl}$ concentration has the net effect of increasing $d D_{\mathrm{T}} / d c$.

Corti and Degiorgio (6) and Rohde and Sackmann (23) used QELSS to obtain $D_{\mathrm{m}}$ for SDS micelles at varying SDS concentrations using background electrolyte concentrations of $0.6-0.1$ and 0.2 to $0 M$, respectively. At low ionic strength $I, d D_{\mathrm{m}} / d c$ is large. As $I$ is increased, $\left(d D_{\mathrm{m}} / d c\right)$ falls; for $I \geq 0.5 M,\left(d D_{\mathrm{m}} / d c\right)$ is negative. These data accord with the qualitative expectation (9) that repulsive interactions enhance $D_{\mathrm{m}}$, while attractive interactions reduce $D_{\mathrm{m}}$.
Corti and Degiorgio interpret their data in terms of intermicellar electrostatic and van der Waals forces. By assuming that the effective charge $Q$ is independent of $c$, and that electrostatic and attractive forces are dominant at 0.1 and $0.4 \mathrm{M} \mathrm{NaCl}$, respectively, values were obtained for $Q$ and the Hamaker constant $A$. Using $Q$ and $A$ to calculate $D_{\mathrm{m}}$ at other salt concentrations, Corti and Degiorgio found good agreement between theory and experiment. Furthermore, their value $Q=37$ is in remarkably good agreement with the value $Q=36.3$ obtained by Stigter and Mysels (24) from electrophoretic data on sodium lauryl sulfate micelles.

Determining $Q$ from electrophoretic data is a somewhat uncertain procedure, but systematic errors in that calculation will tend to parallel systematic errors in the calculation of $Q$ from $D$. Stigter and Mysels found that $Q$ increases with increasing $I$, contrary to the assumption of Corti and Degiorgio that $Q$ is a constant; $A / K_{\mathrm{B}} T$ may therefore be significantly larger than the $\mathbf{1 1 . 3}$ estimated in Ref. (6). One notes that Ref. (6) obtains $Q$ and $A$ from the concentration dependence of $D_{\mathrm{m}}$ at different $I$, not the magnitude of $D_{\mathrm{m}}$, so any dependence of aggregation number $n$ on $I$ does not affect the result. Rohde and Sackmann interpret their $D_{\mathrm{m}}$ values by using the theory of Stephen (25), which relates $D_{\mathrm{m}}$ to the single free-particle diffusion coefficient $D_{0}=K T / f$, the effective particle charge $Z$, and the concentrations of the small ions in solution. Kratohvil (26) has criticized Rohde and Sackmann's results on the grounds that at low ionic strength they find values of $n$ and $Z$ which are much smaller than those reported elsewhere in the literature. I would suggest that the difficulty is not with the experimental data but rather with Stephen's theory which in other cases does not agree with experiment. In particular, Doherty and Benedek (27) studied the diffusion of charged serum albumin in the limit of low ionic strength, where the theory predicts 


$$
D_{\mathrm{m}}=D_{0}(1+\alpha)
$$

and according to theory $\alpha$ equals the protein charge $Z$. As $Z$ was increased from 4 to 18 , Doherty and Benedek found that $\alpha$ increased from 1.1 to 2.3. That is, if $D_{\mathrm{m}}$ and $D_{0}$ are known, the effective charge $\alpha$ determined by [6] is far lower than the real charge $Z$, a finding which qualitatively tends to explain the discrepancies noted by Kratohvil.

\section{MONOMER-MICELLE EQUILIBRIA, MICELLE POLYDISPERSITY, AND QELSS SPECTRA}

Berne and Pecora (28) present the standard calculation of the light-scattering spectrum of reacting species, stressing that there do not appear to be any published QELSS experiments which unambigously determine fast rate-constants. For an ideal solution containing two noninteracting species $A$ and $B$, the average behavior of the concentration fluctuations is given by Berne and Pecora as

$$
\begin{aligned}
& \frac{\partial C_{\mathrm{A}}(\mathbf{q}, t)}{\partial t} \\
& =\left[-D_{\mathrm{A}} q^{2}-k_{\mathrm{A}}\right] C_{\mathrm{A}}(\mathbf{q}, t)+k_{\mathrm{B}} C_{\mathrm{B}}(\mathbf{q}, t), \\
& \frac{\partial C_{\mathrm{B}}(\mathbf{q}, t)}{\partial t} \\
& =\left[-D_{\mathrm{B}} q^{2}-k_{\mathrm{B}}\right] C_{\mathrm{B}}(\mathbf{q}, t)+k_{\mathrm{A}} C_{\mathrm{A}}(\mathbf{q}, t),
\end{aligned}
$$

where subscripts refer to particular species, $C_{i}(\mathbf{q}, t)$ is the $\mathbf{q t h}$ spatial fourier component of the concentration of species $i$, the $D_{i}$ are mutual diffusion coefficients, and the $k_{i}$ are effective rate constants. While the systems which have been studied by QELSS are in thermal equilibrium, light scattering occurs because the concentrations fluctuate around their average values; consequently, the lefthand sides of [7a], [7b] are not zero. The QELSS spectrum corresponding to [7] contains two exponentials whose decay constants are

$$
\begin{aligned}
\Gamma_{ \pm}=\left(D_{\mathrm{A}}+D_{\mathrm{B}}\right) q^{2}+k_{\mathrm{A}} & +k_{\mathrm{B}} \\
\pm\left[\left(D_{\mathrm{A}}-D_{\mathrm{B}}\right) q^{2}+\right. & \left(k_{\mathrm{A}}-k_{\mathrm{B}}\right)^{2} \\
& \left.+4 k_{\mathrm{A}} k_{\mathrm{B}}\right]^{1 / 2}
\end{aligned}
$$

For very slow reactions $\left(D_{i} q^{2} \gg k_{j}\right)$, one finds $\Gamma_{ \pm} \cong D_{\mathrm{A}} q^{2}, D_{\mathrm{B}} q^{2}$. In this limit a local fluctuation in the concentration of either species is relaxed by diffusion well before the chemical reactions can restore local chemical equilibrium. Species A and B diffuse separately as discrete chemical entities. In the fast reaction limit, the spectrum relaxes with the averaged diffusion coefficient $\Gamma=D_{\mathrm{s}} q^{2}$, where

$$
D_{\mathrm{s}}=\left[k_{\mathrm{B}} D_{\mathrm{A}}+k_{\mathrm{A}} D_{\mathrm{B}}\right] /\left(k_{\mathrm{A}}+k_{\mathrm{B}}\right),
$$

which may be understood in terms of the sequence of events which follow a local concentration fluctuation. If $k_{i} \gg D_{j} q^{2}$, the chemical reactions rapidly turn a fluctuation in the concentration of either species into a fluctuation, with local chemical equilibrium, in the total concentration of all the reacting species. The resulting fluctuation in the total concentration is then relaxed by mass diffusion using the concentration-weighted average diffusion coefficient $D_{\mathrm{s}}$. To apply these results, which hold for a two-solutecomponent ideal solution, to the theory of light scattering by micelles, some substantial refinements are necessary. The major differences between a real suspension of SDS micelles and the two-component model of Berne and Pecora include:

(a) The micelle system contains many more than two components, each component having its own diffusion coefficient and light-scattering cross section. The data of Aniansson et al. (1) indicates that the width

$$
\sigma=\left((n-\bar{n})^{2}\right)^{1 / 2}
$$

of the micelle size distribution is typically $20 \%$ of the mean aggregation number $\bar{n}$, so that a suspension near the CMC may contain more than 20 distinct species of micelle as well as the monomer. For a spherical micelle, $D$ scales as $n^{-1 / 3}$. As the solutes are all small by comparison with the wavelength of light, their scattering cross sections scale as $n^{2}$, so scattering by monomers and small oligomers will only be important below or in the immediate vicinity of the CMC. 
(b) Aniansson et al. (1) argue that the kinetic processes which change $n$ may be grouped into two classes. A set of fast processes, with (for SDS micelles) $\tau_{1} \sim 1.6 \times 10^{7}$ $\sec ^{-1}$, change the size of the micelle by loss or gain of a single monomer, thus ensuring dynamic equilibrium within the distribution of micelle sizes around $\bar{n}$. A second set of processes ensures the equilibrium between the monomer concentration and the total number density of micelles. Because the concentration of small aggregates, e.g., decamers, is very low, these latter processes are very slow, with effective rate constants $\tau_{2} \sim 1-500 \mathrm{sec}^{-1}$.

(c) The work of Corti and Degiorgio strongly indicates that intermicellar interactions have a large effect on $D_{\mathrm{m}}$. Since micelles of comparable $n$ are very similar, it necessarily follows (9) that cross-diffusion coefficients are also important, e.g., a gradient in the concentration of micelles of size $n$ will create a flow in the micelles of size $n+3$, etc.

A complete analytic solution of equations corresponding to [7] for a model which included effects (a)-(c) would be rather complicated. For a two-solute-component, nonideal, reacting system, this author has presented an analysis (29). Fortunately, the ratios of the chemical reaction rates to the diffusion time in an SDS micelle system are such as to allow major simplifications in the discussion. Light scattering observes diffusion through distance of a fraction of a light wavelength. For diffusion coefficients of the magnitude reported by Corti and Degiorgio, $\Gamma_{\text {Diff }}=D_{\mathrm{m}} q^{2} \sim 3 \times 10^{4} \mathrm{sec}^{-1}$. One therefore has $\tau_{1} \gg D_{\mathrm{m}} q^{2} \gg \tau_{2}$, so for the two classes of monomer-micelle reactions, an SDS system is in the fast or the slow reaction limit, respectively, relative to diffusion. The qualitative arguments which explain the limiting behaviors of the two-solute model then predict the effect of each class of reaction on the QELSS spectrum. Since $\tau_{1}$ $\gg D_{\mathrm{m}} q^{2}$, a monomer will typically gain or lose monomers many times before it can dif- fuse through $q^{-1}$. $D_{\mathrm{m}}$ will thus be approximately characteristic of the motion of a $(z$-weighted) average micelle, rather than of a micelle of a particular size.

The transformation of micelles into monomers is discussed by Weinheimer et al. (2), who propose that the averaged $D_{\mathrm{m}}$ includes an appreciable component due to monomer diffusion. Their proposal is based on the explicit assumption that micelle formation and destruction are much faster than mutual diffusion, though, as they recognize, the validity of this assumption depends on the distance over which diffusion occurs. While their assumption would appear to be valid for the Taylor dispersion technique, in which diffusion occurs over macroscopic distances, in QELSS measurements $D q^{2} \gg \tau_{2}$. Over times $\sim\left(D_{\mathrm{m}} q^{2}\right)^{-1}$, individual micelles are stable, albeit dynamically fluctuating assemblies; fluctuations in the total micelle number concentration are relaxed by micelle motion but not by the transformations between micelles and monomers. $D_{\mathrm{m}}$ from a QELSS study therefore does not include monomer diffusion terms. As a corollary, $D_{\mathrm{m}}$ values determined by QELSS are only appropriate for diffusion over uista.ices $\leqslant 10^{-4} \mathrm{~cm}$. Over longer distances, the apparent micelle diffusion coefficient will be larger, since over long distances micelles will have enough time to disperse into monomers, diffuse as monomers, and reassemble into micelles.

If any significant set of reactions had $\tau$ $\approx D_{\mathrm{m}} q^{2}$, reactive effects might be apparent in the spectrum. Mazer et al. (30) have searched for a direct effect of micelle-tomicelle reaction kinetics on the QELSS linewidth $\Gamma$, finding that $\Gamma / q^{2}$ is independent of $q$. Since rate coefficients appear in $\Gamma$ as $q$-independent terms, Mazer et al. concluded that only diffusive processes contribute appreciably to $\Gamma$, as expected from the known chemical reaction rates, for which $\tau \neq D_{\mathrm{m}} q^{2}$.

When intermicellar interactions become important, $\Gamma_{1}$ will depend on a complex 
average of the mutual and cross-diffusion coefficients. Weissman (31) treats scattering from a nonideal monomer-oligomer solution, arguing that $\Gamma$ will be dominated by scattering from the largest oligomers. Because of their low concentration, these large oligomers are expected to move at a rate close to that given by their tracer diffusion coefficient. Weissman, however, discusses a nonreacting system whose average aggregation number is small. The effect of fast reactions on a nonideal system seems not to have been examined.

The rapid micelle-micelle equilibria may have a strong effect on the QELSS lineshape. The spectrum $S(\mathbf{q}, t)$ of a polydisperse nonreacting suspension of micelles would be a sum of exponentials with one exponential for each value of $n$. With careful measurement $S(\mathbf{q}, t)$ may meaningfully be fit to the approximate cumulant expansion (32)

$$
S(\mathbf{q}, t)=B+A \exp \left[\sum_{j=1}^{n}(-1)^{n} K_{n} t^{n} / n !\right],
$$

$A$ and $B$ being experimental constants and $K_{j}$ being the $j$ th cumulant. $K_{1}$ is the average diffusion coefficient. $K_{2}$ indicates the range of diffusion coefficients - the level of polydispersity-in the system. If the distribution of diffusion coefficients is Gaussian, the variance $v=K_{2}^{1 / 2} / K_{1}$ is the width of Gaussian.

A fast reaction does not affect $K_{1}$, but may change $v$ substantially. In the fast-reaction limit, all micelles (regardless of their initial $n$ ) diffuse over the period $\left[D_{\mathrm{m}} q^{2}\right]^{-1}$ as average micelles, each with the same diffusion coefficient. As $\tau_{1} /\left(D_{\mathrm{m}} q^{2}\right) \rightarrow \infty$, the spectrum smooths itself into a single exponential, so $v \rightarrow 0$. Corti and Digiorgio (4) report variances $v$ for QELSS spectra of SDS micelles in $0.6 \mathrm{M} \mathrm{NaCl}$, finding $v \sim 0.3-0.5$. The observation of a nonzero $v$ may indicate that the system is not in the fast-reaction limit, i.e., during $\left[D_{\mathrm{m}} q^{2}\right]^{-1}$, the reactions are not fast enough to turn a fluctuation in the concentration of one species into a fluctuation in the total concentration. A partial reduction of $v$ by monomer-micelle reactions will, however, make it very hard to extract information about the form of the aggregation number distribution from the lineshape.

Weinheimer et al. (2) also suggest that the dynamic nature of a micelle may lead to some unusual effects in QELSS measurements. One such phenomenon, as recently proposed by this author, is reactive diffusion (33), in which the monomer-micelle reactions move the micelles. In order for the monomer and micelle to bind, or for a monomer to be ejected by a micelle, the centers of mass of monomer and micelle must be displaced relative to each other by a distance on the order of a monomer length. The micelles are not infinitely massive, so during each reaction a part of the monomermicelle displacement is due to micelle motion. As reactions occur in a solution rather than a vacuum, the part of the total displacement which is due to micelle motion will depend on the relative drag coefficients, and not the relative masses, of micelle and monomer. When a micelle gains and loses monomers, it performs a random walk at a speed determined by kinetic rate coefficients, as well as by hydrodynamics. As shown by this author, this process increases the translational diffusion coefficient of a micelle, but is indistinguishable (at least by QELSS studies) from conventional Brownian motion. Numerical examination indicates that reactive diffusion is a $1 \%$ correction for SDS micelles, but represents a $35 \%$ effect in the motion of sodium hexyl sulfate micelles.

\section{SUMMARY}

The modern treatment of macroparticle diffusion in terms of tracer and mutual diffusion coefficients is adequate to interpret reported values for the diffusion coefficients of SDS micelles. The differences between the data of Stigter et al. (2) and data obtained with QELSS (4-6) may reasonably be ascribed to the differing effect of intermicel- 
lar interactions on $D_{\mathrm{m}}$ and $D_{\mathrm{T}}$, as shown by the calculations of Mazo (15) and Corti and Degiorgio (6). The discrepancies with the results of Rohde and Sackmann (23) can be interpreted as arising from the theory used to interpret their measurements, rather than from the measurements themselves.

The possible influence of monomermicelle equilibria on QELSS spectra is discussed at length, using a realistic model (1) for the reaction kinetics. To good approximation, the micelle number density is conserved on the QELSS time scale. However, the model of Aniansson et al. indicates that the fluctuation in the aggregation number are very fast, so that QELSS measures the motion of an average micelle, and not the separate motions of micelles of different sizes. A simple interpretation of the nonexponential QELSS line shape as arising from the micelle size distribution is therefore not possible.

\section{ACKNOWLEDGMENT}

The partial support of this work by the National Science Foundation under Grant CHE 79-20389 is gratefully acknowledged.

\section{REFERENCES}

1. Aniansson, E. A. G., Wall, S. N., Almgren, M., Hoffmann, H., Kielmann, I., Ulbricht, W., Zana, R., Lang, J., and Tondre, C., J. Phys. Chem. 80, 905 (1976).

2. Weinheimer, R. M., Evans, D. F., and Cussler, E. L., J. Colloid Interface Sci. 80, 357 (1981).

3. Stigter, D., Williams, R. J., and Mysels, K. J., J. Phys. Chem. 59, 330 (1955).

4. Corti, M., and Degiorgio, V., Chem. Phys. Lett. 53, 237 (1978).

5. Corti, M., and Degiorgio, V., in "Solution Chem- istry of Surfactants"' (K. L. Mittal, Ed.), Vol. 1. Plenum, New York, 1979.

6. Corti, M., and Degiorgio, V., J. Phys. Chem. 85, 711 (1981).

7. Chen, S.-H, and Yip, S., Editors, "Spectroscopy in Biology and Chemistry Neutron, X-ray, Laser." Academic Press, New York, 1979.

8. Phillies, G. D. J., J. Chem. Phys. 60, 983 (1974).

9. Phillies, G. D. J., J. Chem. Phys. 60, 976 (1974).

10. Hall, R. S., Oh, Y. S., and Johnson, C. S., J. Phys. Chem. 84, 756 (1980).

11. Ackerson, B. J., J. Chem. Phys. 69, 684 (1978).

12. Dieterich, W., and Peschel, I., Physica 95A, 208 (1979).

13. Marqusee, J. A., and Deutch, J. M., J. Chem. Phys. 73, 5397 (1980).

14. Phillies, G. D. J., J. Chem. Phys. 74, 2436 (1981).

15. Mazo, R. M., J. Chem. Phys. 43, 2873 (1965).

16. Gros, G., Biophys. J. 22, 453 (1978).

17. Jones, C. R., Johnson, C. S., Jr., and Penniston, J. T., Biopolymers 17, 1581 (1978).

18. Phillies, G. D. J., Benedek, G. B., and Mazer, N. M., J. Chem. Phys. 65, 1883 (1976).

19. Phillies, G. D. J., and Wills, P. R., J. Chem. Phys., 75, 508 (1981).

20. Felderhof, B. U., J. Phys. A. Math Gen. 11, 929 (1978).

21. Batchelor, G. K., J. Fluid. Mech. 74, 1 (1976).

22. Weissman, M. B., and Ware, B. R., J. Chem. Phys. 68, 5069 (1978).

23. Rohde, A., and Sackmann, E., J. Colloid Interface Sci. 70, 494 (1979).

24. Stigter, D., and Mysels, K. J., J. Phys. Chem. 59, 45 (1955).

25. Stephen, M. J., J. Chem. Phys. 55, 3878 (1971).

26. Kratohvil, J. P., J. Colloid Interface Sci. 75, 271 (1980).

27. Doherty, P. A., and Benedek, G. B., J. Chem. Phys. 61, 5426 (1974).

28. Berne, B., and Pecora, R., "Dynamic Light Scattering." Wiley, New York, 1976.

29. Phillies, G. D. J., J. Chem. Phys. 59, 2613 (1973).

30. Mazer, N. A., Benedek, G. B., and Carey, M. C., J. Phys. Chem. 80, 1075 (1976).

31. Weissman, M. B., J. Chem. Phys. 72, 231 (1980).

32. Koppel, D. E., J. Chem. Phys. 57, 4814 (1972).

33. Phillies, G. D. J., J. Phys. Chem., in press. 